\title{
II Bicentenario Leopardiano in Spagna. Bilancio e bibliografia
}

M aría de las N ieves M uñiz M uñiz

L'occasione fornita dal Bicentenario della nascita di Giacomo Leopardi (Recanati 1798 - N apoli 1937) ha certamente stimolato, per ovvie ragioni, le iniziative editoriali, ma ha fatto anche affiorare un revival latente nell'humus culturale spagnolo, specie per quel che riguarda poeti neoromantici 0 «cardarelliani» (Sánchez Rosillo, Colinas), e in generale quelli che - al di là delle tendenze segnalate - fanno capo a Luis C ernuda, il meno «puro» 0, se vogliamo, il più tormentato e autobiografico fra i poeti del '27, già contaminatore di Leopardi edi H ölderlin.

D 'altra parte, il progressivo affermarsi di un'italianistica universitaria dopo I'instaurazione della democrazia ha creato le condizioni necessarie per promuovere convegni di studi impensabili in passato, oltre che per pubblicare edizioni di ampio ed originale respiro. Certo, i risultati sono stati disuguali: accanto a lavori seri o quanto meno dignitosi, sono apparsi prodotti improvvisati, banali e persino deleterei. Lo spazio appare ancora dominato dall'approccio occasionale da amateur, mentre nei contributi dei Convegni si avverte ancora una spartizione di campi fra specialisti stranieri dediti a studiare Leopardi per se, e filologi o poeti spagnoli interessati alla ricezione dello scrittore in Spagna. Tuttavia, le eccezioni alla regola non mancano e raggiungono pure a volte un ottimo livello che porta dell'aria fresca nell'atmosfera un po' stagnante del leopardismo per cosi dire «ufficiale». Di questa apertura di orizzonti e di frontiere è indizio significativo una Tesi di D ottorato sui Canti discussa presso I'U niversitat de Barcelona l'anno del Bicentenario, il cui autore poté a suo tempo seguire un seminario presso il Centro N azionale di Studi Leopardiani, per poi trovare all'università di origine un clima propizio per proseguire gli studi. La risposta spagnola alla sfida del '98 è stata in ogni caso massiccia. Per darne dovuto conto, offriamo qui di seguito una bibliografia analitica dalla quale restano soltanto esclusi gli interventi giornalistici (a meno che non contengano traduzioni inedite), le recensioni equalche incontro celebrativo i cui Atti non siano stati annunciati. 


\section{A. TRADUZIONI}

\section{A1. Traduzioni dei CANTI}

Antología poética, a cura di Eloy Sán chez Rosillo, Valencia, PreTextos, «C ruz del Sur», 1998, 197 p.

[Trad. castigliana in versi di diciotto canti più un abbozzo; tendenzialmente rispettosa del senso e del ritmo: II passero solitario, L'infinito, La sera del di di festa, Alla luna, La vita solitaria, A Silvia, Le ricordanze, Canto notturno, La quiete dopo la tempesta, II sabato del villaggio, II pensiero dominante, Amoree M orte, A se stesso, Aspasia, Sopra un basso rilievo antico sepolcrale, II tramonto della luna, La ginestra, Imitazione, Scherzo, II canto della fanciulla. Breve prefazione (p. 9-15); in appendice, breve notizia informativa su ogni poesia tradotta].

Cantos, a cura di José Luis Bern AL, Granada, La Veleta, 1998, 309 p.

[Trad. castigliana dei $C$ anti, escluse le imitazioni ei frammenti, realizzata in M essico; testo a fronte. Breve premessa del traduttore e di M aria Pia Lombardi (p. 9-12)].

Cantos escogidos, a cura di Luis M ARTín Ez M ERLO, M adrid, H iperión, 1998, 205 p.

[Scelta di 21 canti tradotti in castigliano con testo a fronte e breve premessa (p. 9-11): All'Italia, A un vincitore ne pallone, Alla primavera, U Itimo canto di Saffo, II passero solitario, L'infinito, La sera del di di festa, Alla luna, II sogno, Alla sua donna, A Silvia, Le ricordanze, Canto notturno, La qui ete dopo la tempesta, II sabato de villaggio, Amore e M orte, A se stesso, Sopra un basso rilievo antico sepolcrale, II tramonto della luna, La ginestra, Scherzo].

Cantos, trad. in galiziano di 20 canti (prevalentemente idilli) a cura di Suso PEn SADo, A Coruña-G aliza, Espiral M aior, 1998, 145 p.

[Traduzione in galiziano con testo a fronte di 20 canti: U Itimo canto di Saffo, II passe ro solitario, L'infinito, La sera del di di festa, II sogno, La vita solitaria, Alla sua donna, A Silvia, Le ricordanze, Canto notturno, La quiete dopo la tempesta, II sabato de villaggio, II pensiero dominante, Amore e M orte, Aspasia, Sopra un basso rilievo antico sepolcrale, Sopra il ritratto di una bella donna, Palinodia, II tramonto della luna, La ginestra. Breve $N$ ota del Traduttore con al cune inesattezze sulla vita e sull'opera di L.].

Cantos, a cura di N . M uÑIZ M uÑIZ, M adrid, Cátedra, «Letras U niversales», 1998, $950 \mathrm{p}$.

[Traduzione integrale dei Canti in versi, con testo a fronte: Introduzione, Indice di Autori citati da L. e presenti in apparato, ampia bibliografia sui singoli canti e commento (quest'ultimo alle p. 528-950). La traduzione, che tenta di riprodurre il ritmo, le assonanze ele rime più significative, ha ricevuto il Premio Città di M onselice «D iego Valeri» e quello del M inistero degli Affari Esteri italiano; I'Introduzione interpreta sinteticamente il libro attraverso il suo farsi el'opera complessiva di L. attraverso lo scontro con l'orizzonte d'attesa dei contemporanei e dei posteri (compreso il capitolo su Leopardi in Spagna). O gni canto è preceduto da un cappello di ampie proporzioni coronato da una scheda filologica e metrica. Segue in fondo al volume un fitto apparato di note esegetiche che costituiscono una lettura interlineare dei $\mathrm{C}$ anti alla luce della Ioro intertestualità (p. 528-948). II volume contiene, purtroppo, un numero importante di refusi (particolarmente fastidiosa l'applicazione meccanica della divisione sillabica spagnola all'italiano)]. ${ }^{1}$

1. In attesa di apportare le opportune correzioni alla seconda edizione dell'opera, ci si limita qui a sanare alcuni errori fra i più nocivi:

p. 17, riga 19: Introducción: dove dice «no me fuese lícito» si legga: «me fuese lícito». 


\section{A2. Ristampe e varia}

Poesía y prosa, a cura di Antonio Co LIN AS, Barcellona, Círculo de Lectores, 1998 (ristampa corretta della 1aㅡ ed. M adrid, Alfaguara,1980).

Poemas elegi dos, a cura di M ilagros ARIZM EN DI, M adrid, Adonais, 1999.

[Riproduzione antologica di traduzioni di varia mano e provenienza: All'I talia (Loreto Busquets), U Itimo canto di Saffo (D iego N avarro), II Passero solitario (Juan Luis Estelrich), L'infinito (Tomás M orales), La sera del di di festa (Jerónimo Rosselló), La vita solitaria (Eloy Sánchez Rosillo), A Silvia (Antonio Colinas), La qui ete dopo la tempe sta (Juan O'N eille), Amore e M orte (Eloy Sánchez Rosillo), La ginestra (M iguel de Unamuno)].

\section{A3. Traduzioni di singoli canti e dei Paralipomeni}

El viatge del comte Escurafons a l'illa del'infern, a cura di Lola BADIA, fasc. 45 di Senhal, Girona, autunno 1998, 12 p., Edizione tipograficamente curata da Patrick G ifreu, Enric Prat e Pep Vila con la collaborazione della «Llibreria 22» e del Comune di Girona.

[Traduzione in prosa catalana di Paralipomeni, VII, 24-51; preceduta da una presentazione dell'opera dove si sottolinea il rovesciamento dissacrante delle fonti virgiliana e dantesca (p. 2-3); tiratura in 200 esemplari con ill. tratte dalla M atèria mèdica di D ioscoride, a cura di Andrés LAGUnA, Salamanca 1570].

Canti. Traduzioni di D uque Amusco, Borfoot, Bonnefoy, Elliot, Enzensberger, $\mathrm{H}$ utche son, Jaccottet, Lynch, M uñiz M uñiz, Pierpoint, Purin, Singh, Vaghenàs, Vol'tskaja, Centro M ondiale della poesia e della cultura «G iacomo Leopardi» e Centro di Poesia C ontemporanea, Università di Bologna, a cura di Rolando G ARBU GLIA e Stefano M ALDINI, Bologna, 1999, 163 p.

[Versioni con testo a fronte. Allep. 11-15, El infinito e A ś mismo traduzioni in castigliano del poeta Alejandro D uque Amusco (quella dell'Infinito fatta per I'occasione; quella di A se stesso risalente agli anni Settanta e inedita); alle p. 105-129, El infinito e La retama o la flor del desierto, riproduce la traduzione dell'Infinito e della Ginestra a cura di N . Muñiz Muñiz].

p. 137: Ad Angelo M ai, v. 90: dove dice «al chiquillo parece que no al sabio», si legga: «no parece al chiquillo como al sabio».

p. 221: Il passero solitario, v. 8: dove dice «mugir las manadas», si legga: «mugir manadas».

p. 351: Canto notturno, v. 43: dove dice «lo empiezan», si legga: «o empieza».

p. 407, riga 1: dove dice «tres grandes estrofas (la segunda... )» si legga: «cuatro grandes estrofas (la segunda y la tercera... )».

p. 523, riga 13: D ello stesso (cappello introduttivo): dove dice «si priores M aeonius tenet», si legga: «non, si priores M aeonius tenet».

p. 573, riga 19: Ad Angelo M ai, nota ai w. 159-162: dove dice «apunte del Zib.», si legga: «apunte del Zib., 2454».

p. 588, riga 42-44: A un vincitore nel pallone, $\mathrm{N}$ ota al v. 40: dove dice «"M ientras invocamos esas sombras magnánimas, nuestros enemigos pisotean sus sepulcros. E verrà forse giorno che noi... sarem fatti simili agli schiavi", así comentado en Zib., 205: "Y vendrá quizá un día [E verrà forse giorno] que perdiendo las riquezas"», si legga: «M ientras invocamos esas sombras magnánimas, nuestros enemigos pisotean sus sepulcros. "Y vendrá quizá un día [E verrà forse gi orno] que perdiendo las riquezas..." (cit. en Zib., 58)».

p. 668, riga 13: L'Infinito (nota al v. 7): dove dice «Aen., VII, 144», si legga: «Aen., VII, 646». 
L'infinit, in N arcís C o M ADIRA, Escriure per sempre, El País. Catalunya, «Q uadern», giovedì 12 marzo 1998, p. 2.

[Traduzione in catalano dell'Infinito a cura del poeta $\mathrm{N}$ arcís Comadira, autore dell'articolo; la versione presenta varianti rispetto a quella già pubblicata nell'antologia Poe sia italiana, Barcellona, Edicions 62, 1985, p. 218, e costituisce un assaggio della traduzione completa dei Canti che il poeta catalano prepara da tempo].

El infinito, in Raffaele PINTO, «'Infinito y el nihilismo positivo de G iacomo Leopardi», Er. Revista de Filosofía, n. 24-25, 1999, p. 93-115.

[C ontiene la traduzione in lingua castigliana dell'Infinito a cura dell'autore dell'articolo; cfr. infra sez. B4].

\section{A4: Prosa: 0 perette, Pensieri, D iscorsi, Zibaldone}

O bretes morals, trad. in catalano delle 0 perette a cura di Rossend ARQUÉs, Barcellona, D estino 1996, $340 \mathrm{p}$.

[Traduzione in catalano delle 0 perettemorali. $N$ ota al testo, $\mathrm{N}$ ota alla traduzione, Bibliografia, Postfazione e note a cura del traduttore. La premessa, Leopardi filòsof, riproduce un testo di Giorgio Colli pubblicato in precedenza].

Pensamientos, a cura di César Palm A, Valencia, Pre-Textos, 1998, 174 p.

[Trad. in castigliano dei $\mathrm{CxI}$ Pensieri. Breve prefazione sotto forma di un dialogo immaginario con il poeta morto, che si confessa più affine a Schopenhauer che a N ietzsche e dichiara le proprie preferenze per Luciano, Borges, Bayle, Luis M aristany (p. 9-17)].

D iscurso de un italiano en torno a la poeśa romántica, a cura di Carmelo VERA SAURA, Valencia, Pre-textos, 1998, 302 p.

[Prima traduzione in castigliano del $\mathrm{D}$ iscorso di un italiano intorno alla poesia romantica. Contiene un'ampia Introduzione informativa e note pure di taglio informativo].

Zibaldone dels pensaments, a cura di Assumpta C AM PS, Barcellona, C olumna-L'Albí, 1998, $611 \mathrm{p}$.

[Prima traduzione in catalano di un'ampia scelta dallo Zibaldone col contributo del Centro N azionale di Studi Leopardiani; N ota di Franco Foschi e Introduzione della curatrice (p. 11-20). Gravissimi difetti annullano l'utilità dell'impresa editoriale: I'Introduzione è, pur nella sua modestia divulgatrice, cosparsa di calchi non virgolettati e male interpretati dalla prefazione di Giuseppe Pacella all'edizione critica dello Zibaldone (M ilano, G arzanti, 1991); le note informative sono pure cal cate su quelle di Pacella; il rinvio alla pagina dell'autografo si omette spesso provocando disorientamento nel lettore; il danno è completato dai numerosi sventramenti del testo, non sempre segnalati; la traduzione, infine, appesantisce e appiattisce nel contempo la lingua leopardiana e incorre in errori di comprensione tanto numerosi quanto stravolgenti (spesso sotto forma di quid pro quo). Paradossale risulta, dunque, il giudizio negativo emesso dalla traduttrice sullo stile del «maggior prosatore del secolo» (parole di $\mathrm{N}$ ietzsche), quando afferma nell'Introducció che «la redacció» originale è «descurada i reiterativa». La versione ha ottenuto il premio «Serra d'O r» 1998].

\section{A5. Traduzioni di singoli pensieri dallo Zibaldone}

Pensamientos, a cura di Agustín IzQuierdo, in Revista de 0 ccidente, Madrid, ottobre 1998, p. 142-48.

[Trad. castigliana di 26 frammenti di varie epoche, senza indicazione né di data né di pagina. La scelta è preceduta da un breve profilo di Leopardi filosofo in chiave nietzscheana: Leopardi (1798-1837). Vivir sin ilusiones (cfr. sezione B)]. 
Leopardi, una vida hecha de palabras, a cura di Josep M ASSOT, in La Vanguardia, Barcellona, 29 giugno 1998.

[Traduzione in castigliano di 16 frammenti zibaldoniani, senza indicazione né di data né di pagina. La scelta è preceduta da una breve notizia sull'edizione critica a cura di Pacella e da un profilo del poeta tendente a confutare la tesi della misantropia e a propugnare la sua importanza come pensatore. L'interpretazione in chiave nietzscheano-decadente emerge in finis: «un mundo desolado y soportable sólo por la imaginación, el sueño, la droga o la ilusión, un concepto que, en un vértigo de ecos, une, por ejemplo, las teorías brahmánicas con Baudelaire o el pensamiento existencial de este siglo». In compenso, sottolinea la novità della prosa leopardiana].

La poeśa en la era de la bolsa y el periodismo, a cura di Rossend ARQUÉs, in Archipiélago, n. 37, estate 1999, p. 79-83.

[Traduzione castigliana di sei frammenti intorno all'impraticabilità della poesia nell'età della tecnica: quattro dallo Zibaldone (Zib. 2944-46, 3241-42, 4418, 4497), due dall'epistolario (lettera a G. M elchiorri del 5 marzo 1824; lettera a G. Giordani del 24 luglio 1828). In una nota di commento a pie' di pagina il traduttore sottolinea il superamento «eroico» del problema nel 1828 el'original ità della soluzione praticata nella «Ginestra»].

\section{B. STUDI}

\section{B1. Atti di Convegni e Volumi collettivi}

Giacomo L eopardi. Poesia, Pensiero e Ricezione, Atti del Convegno Internazionale di Barcellona organizzato dall'U niversitat de Barcelona, dall'U niversitat de Girona e dall'U niversitat Autònoma de Barcelona in collaborazione con la Giunta N azionale Leopardiana, il C entro N azionale di Studi Leopardiani el'Istituto Italiano di C ultura di Barcellona (marzo 1998), a cura di M aría de las N ieves M UÑ IZ M UÑ IZ con la collaborazione di Francisco Am ELLA e Francesco ARD o LIN O, Leonforte, Insula, 2000, 414 p.

[«Introduzione» di N ieves M uñiz. PARTE I. POESIA / PEN SIERO: D omenico De RoberTIS, «La verità della Ginestra»; Antonio PRETE, «Apparenza, finitudine, leggerezza: sulle $O$ perette morali»; Lola BADIA, «Lelusinghe dell'amore romantico nei Canti enelle 0 pe rette»; Francisco Am ELLA VeLA, «l filo del tramonto nei Canti»; Bianca LoTITO PRIM ICERI, «''esattezza del vago»; Franco BRIOSCHI, «Per un Leopardi empirista»; Anna D OLFI, «T ipologie del pensiero e forme dell a soggettività nello Zibaldones; M ario Andrea RIGO$\mathrm{NI}$, «Sul nulla e sulla negazione nel pensiero di Leopardi»; Raffaele PIN TO, «I desiderio nello Zibaldones; M aria PERT ILE, «Le parole ele cose: sul "Vocabolario Universale Europeo"»; Enrico G HIDETTI, Alle origini del sentimento poetico leopardiano; Alejandro PÉREZ VIDAL, «"Bellezze parziali". Leopardi e la poetica romentica della sátira». PARTE II. RICEZIONE: Giovanni ALBERTOCCHI, « Leopardi e M anzoni nella scia del 1827»; Laura M ELOSI, «C onversare nella distanza. Sul carteggio Leopardi-V ieusseuX»; Elisabetta BENUC$\mathrm{Cl}$, «Leopardi eil carteggio con Vieusseux. Le lettere da Recanati (novembre 1828-marzo 1830)»; Gilberto Lo ARDI, «Un ascolto "sviato": il Leopardi di M ontale»; Piero D AL BON, «I l leopardismo nei Frammenti Lirici di Clemente Rebora»; Laura BARILE, «La lettera e lo spirito. Su alcune traduzioni da Leopardi di Guillén, Laforgue e Lowell». RICEZIONE IN SPAGNA: M aría de las N ieves M UÑ IZ, «Pensiero vs Poesia: Leopardi in Spagna»; Rossend ARQUÉS, «Leopardi nella Catalogna del N ovecento»; Cesáreo CaLvo RIGUAL, «LaS traducciones de los Cantos en la Antología de Estel rich (1889)»; D onatella G AGLIARDI, «Albert Aldrich traduttore dei Pensieri di Leopardi»; Gabriella GAVAGNIN, «La vita solitaria nelle versioni di Josep C arner e di Tomàs G arcés»; Cristina M ARCH ISIO, «U na tra 
duzione dell'Infinito e altro Leopardi in Galizia (il caso di Antón Losada D iéguez)»; Anna GIORDAN 0, «Presenza di Leopardi in alcuni epistolari di scrittori spagnoli: M iquel Costa i Llobera, Antoni Rubió i Lluch, Josep C arner»; Francesco ARD o LIN 0, «Leopardi in M aragall»; Eduard VILELLA, «Lo Zibaldone secondo J osep Pla». IN DICE DEI NOM I].2

M entre nel mondo si favelli o scriva. Giacomo Leopardi en el II centenario de su nacimiento (1798-1998), M adrid, Universidad Complutense, D epartamento de Filología Italiana, 1998, 369 p.

[«Premisa» di Cristina Barbolan I; Parte I. El Pen sam iento: Aurora Conde, «Algo más sobre las $O$ perette morali »; C arlo FERRUCCI , «Leopardi, o la radicalità dell'interezza»; Jacobo M UÑ OZ, «La materia última de la vida»; PARTE II. TEM AS Y GÉNEROS: Cristina BARBO LAN I, «U na lettura della Telesilla leopardiana»; M irella M AROTTA, «EI tema del viaje en la obra teórica de G iacomo Leopardi»; Elisa M ARTín EZ GARRID O, «All'I talia de G iacomo Leopardi. De nuevo sobre la influencia del Romanticismo en el discurso político dela pasión en Italia»; Enrique O Tón SOBRIN O, «La visita en sueños (a propósito de Propercio IV, 7 e ll sogno de Leopardi»; Rosario SCRIM IERI, «Leopardi y La pietra lunare de Tommaso Landolfi»; PARTE III. RECEPCIÓN: M aría de los Ángeles ARCE, « orge Guillén ante la luna de Leopardi (la medida de un homenaje)»; Trinidad BLAN CO, «C alas leopardianas»; G loria G ALLI, «Presencia de Leopardi en Argentina»; Vicente G O NZÁLEZ M ARTín, «Giacomo Leopardi en Carmen deBurgos»; Elena LoSADA SO LER, «D os voces de la tragedia romántica: Antero de $Q$ uental y Giacomo Leopardi». PARTE IV. CREACIÓN : Antonio Colinas, «Leopardi y mis "Leopardis"»; M iguel Ángel Cuevas, «O stia, Tabor (G iacomo Leopardi, Pier Paolo Pasolini)»; Luis Antonio DE VILLEN A, «U na carta no enviada que Giacomo Leopardi escribió a Lord Byron, que por entonces vivía en Venecia»].

Relaciones culturales entre I talia y España, VI Encuentro entre las U niversidades de M acerata y Alicante (noviembre, 1998), D epartamento de Filología Española, Universidad de Alicante, a cura di Ángel L. Prieto de Paula e Juan A. Ríos, Alicante, Aguaclara, 1999, p. 9-95.

[Luigi BANFI, «Pensieri sulla Spagna nello Zibaldone»; Giulia M ASTRANGELO, «ntorno ad alcune riflessioni del Leopardi nello Zibaldone»; Pedro Luis LADRÓN DE GUEVARA, «Leopardi y Recanati como motivo poético en los poetas españoles»; Antonio M OREN O, «Leopardi: la vida solitaria (El espacio último de los Cantos)»; Ángel Luis PRIETO DE PAULA, «La sera del di di festa: el destierro en la tierra, o la paradoja de un locus leopardiano»; Belén T EJERIN A, «El leopardismo de osé Alcalá Galiano y Fernández de las Peñas, Conde de Torrijos»)].

O sservazioni: alle p. 95-141 seguono altri tre saggi su argomenti non leopardiani.

\section{B2. Tesi di dottorato}

Francisco Am elLA Vela, Giacomo Leopardi: «Il fuggitivo sol». El motivo del ocaso y el problema dela ordenación de los «C anti», $286 \mathrm{p}$.

[Tesi di D ottorato discussa presso l'U niversità di Barcellona nel giugno del 1998. «Pre mio Extraordinario» conferito dall'U niversitat de Barcelona. Propone una lettura dei Canti come «canzoniere» articolato da saldi fili conduttori quali la istanza enunciatrice dell'«io» e alcuni temi sotterranei: nella fattispecie il «tramonto», utilizzato anche ai fini della datazione del Passero solitario. È la prima tesi sul poeta in Spagna].

2. A pagina VIII si allude alla partecipazione di Rosario Scrimieri alla tavola rotonda su «Leopardi in Spagna oggi»; in realtà si doveva scrivere Cristina Barbolani. 


\section{B3. Articoli su rivista}

Agustín IzQuIERD 0, «Leopardi (1798-1837). Vivir sin ilusiones», Revista de 0 ccidente, M adrid, ottobre 1998, p. 135-141.

[Breve profilo di Leopardi filosofo in chiave nietzscheana, seguito dalla traduzione di una scelta di frammenti zibaldoniani; cfr. supra].

Raffaele PINTO, «'infinito y el nihilismo positivo de Giacomo Leopardi», Er. Revista deFilosofía, n. 24-25, 1999, p. 93-115.

[Traduce ed analizza L'infinito vedendo nella sacralizzazione della finitudine la modernità del componimento].

M aría de las N ieves M UÑ Iz M UÑ Iz (in collaborazione con Lola Badia), «D e G iacomo Leopardi al Cottolengo o el trànsit de la lucidesa a l'estultícia», Els M arges, n. 65, dicembre 1999, p. 85-111.

[Analizza gli errori della traduzione catalana dello Zibaldone a cura di A. Camps (cfr. supra A4), aggiungendo in appendice un Dizionario alfabetico di quid pro quo].

\section{Bibliografie}

Giacomo Leopardi. Poesia/Pensiero e Ricezione. La ricezione in Spagna. Tradurre Leopardi, a cura di M. N . M UÑ IZ M UÑ IZ, Barcellona, PPU , ISBN 844770636 2, 1998, $105 \mathrm{p}$.

[O puscolo pubblicato in occasione del Bicentenario leopardiano ad uso dei partecipanti al Convegno Internazionale di Barcellona: Giacomo Leopardi: Poesia/Pensiero e Ricezione (marzo 1998). Riproduce traduzioni storiche di testi leopardiani (Sardà, M aseres, Garcés, C arner, M enéndez Pelayo, U namuno, Juan Ramón Jiménez, Jorge Guillén, Aldrich, Pla), e contiene una ampia Bibliografia leopardiana spagnola (Traduzioni, Studi, Imitazioni) a cura di N. M uñiz M uñiz (p. 70-105)]. 